Dr. Isidro Catela

Profesor de la Universidad Francisco de Vitoria

@ i.catela.proł@ufv.es
Recibido / Received 4 de enero de 2019

- Aceptado / Acepted 1 de febrero de 2019

- Páginas / Pages De la 61 a la 80

- ISSN: 2531-0054

\title{
Fundamentos éticos del derecho a la desconexión digital
}

\section{Ethical foundations of the right to digital disconeection}

El Gobierno español está preparando una nueva Ley de Protección de Datos en la que se incluyen de forma novedosa una serie de derechos digitales, entre los que se encuentra el conocido como derecho a la desconexión. En diálogo con la ética clásica, el artículo desvela algunos de los fundamentos prejurídicos en los que se asienta el citado derecho, partiendo de un análisis del contexto que hunde sus raíces en lo que el filósofo Byung Chul-Han llama «la sociedad del cansancio».

PALABRAS CLAVE: derecho, ética, desconexión digital, razón abierta, virtudes, vicios, sociedad del cansancio.

\section{La sociedad hiperconectada}

\subsection{Sociedad del trabajo, rendimiento e hiperconexión: las aportaciones de Byung Chul-Han}

La nueva legislación de protección de datos, que adaptará el ordenamiento jurídico español al nuevo reglamento europeo sobre la materia, está en las últimas fases de su tramitación parlamentaria. Con apreciable consenso, todo hace prever que en breve tendremos una nuevo texto en España que regulará una serie de derechos digitales (según la propia denominación que aparece en el Informe de la Ponencia del Proyecto de Ley), como el derecho al olvido o el derecho a la desconexión digital por parte de los trabajadores. 
¿A qué preocupación social (antropológica y ética) responde esta propuesta? ¿No deberíamos alegrarnos de que nuestros hijos vinieran, en pleno siglo XXI, con un pan digital debajo del brazo? ¿Qué voces de alarma están sonando? ¿Están justificadas? ¿No será, una vez más, una suerte de actitud ludita, que a modo de la sostenida por el conocido movimiento artesanal inglés del siglo xIx, la esté emprendiendo contra las máquinas al considerarlas enemigas del progreso y amenazas para su propio trabajo?

En este sentido, la reflexión sobre la nueva legislación ha de partir del análisis del humus sobre el que nace y trata de asentarse. No parece casualidad que empresas como lkea, la multinacional sueca de los muebles, o el licor Ruavieja, por citar solo dos recientes ejemplos, estén tratando de posicionar su imagen de marca en torno a una misma idea: la de que desarrollaremos mejor el ideal de vida buena al que estamos llamados cuanta más cabeza pongamos en el uso de la tecnología, y por lo tanto más nos alejemos del fantasma de la adicción digital, que amenaza con convertirnos en consumidores consumidos ${ }^{1}$. En una sociedad que ensalza el simulacro y la representación, que se caracteriza por la omnipresencia de las pantallas (Catela, 2012), pareciera como si ahora tuviéramos que desconectar para volver a conectar con la realidad (Catela, 2018) y aprehenderla como merece.

El filósofo surcoreano, afincado en Alemania, Byung Chul Han, está tratando en los últimos años de alumbrar algún criterio sólido para comprender esta sociedad, la sociedad líquida (Bauman, 2007) que nos ha tocado vivir. Aristóteles nos enseña que el hombre es, por naturaleza, un animal político. Posee, de modo exclusivo, y frente a los demás animales, el sentido de lo bueno y lo malo, lo justo y lo injusto, y las demás apreciaciones. Es, precisamente, la participación comunitaria en estas la que funda la ciudad (polis), que los griegos entendían como unidad política suprema, y que es la que buscará el fin supremo que implica a la totalidad y que no es otro que la felicidad de todos los ciudadanos. Para Han, sin embargo, el hombre contemporáneo es un animal gregario más, que se sitúa en lo que él llama enjambre digital, un lugar cuyo centro neurálgico son las redes sociales, que a su vez erosionan el espacio público y que agudizan el aislamiento del hombre (Han, 2014, 75). Este enjambre es una comunidad frecuentemente indignada, pero la indignación por sí misma no crea comunidad alguna, porque aglutina multitudes volátiles, poco firmes, que crecen súbitamente y se dispersan con la misma rapidez; un entorno que no desea crear ninguna comunidad, ningún nosotros, y que son más bien una concentración sin congregación, una multitud sin interioridad alguna. Precisamente a este respecto, la obra más significativa de Han es la que lleva por título En el enjambre, donde expone que estas olas de indignación son muy eficientes para movilizar y aglutinar la atención en un primer momento, pero que son inapropiadas para configurar el discurso y el espacio público. La llíada comienza con la palabra ira, nos recuerda Han. Es un canto de la ira, que constituye la primera narración de la cultura occidental. «La ira puede cantarse aquí, porque soporta, estructura, anima, vivifica. Esta ira es narrativa, épica, porque produce determinadas acciones [...]. La indignación digital, en cambio, no puede cantarse. Es un estado afectivo que no desarrolla ninguna fuerza podero-

1. La campaña «Familiarizados» de lkea puede verse en https://www.youtube.com/watch?v=grTQHDlcl2w\&t=4s y la denominada «Tenemos que vernos más», de Ruavieja, en https://www.youtube.com/resultssearch_query=tenemos+que+vernos+m\%C3\%A1s+anuncio+ruavieja+2018. Ambas fueron lanzadas en las semanas previas a la Navidad de 2018. 
sa de acción» (Han, 2013, 22). Por todo ello, no engendra ningún futuro, sino que es, más bien, causa de saturación y hastío. Es lo que el propio Han en La sociedad del cansancio llama violencia neuronal: «Toda época tiene sus enfermedades emblemáticas. Las propias del siglo xxı son patologías neuronales tales como el déficit de atención con hiperactividad, el trastorno límite de personalidad o el síndrome de desgaste ocupacional (SDO)» (Han, 2012, 11). No hace falta ahondar demasiado en la relación que existe entre este último síndrome y el objeto de estudio que nos ocupa. Así, la sociedad del trabajo constante, del rendimiento, no sería, en sentido estricto, una sociedad libre, sino una sociedad que, al producir nuevas y constantes obligaciones, convierte al individuo en esclavo del trabajo, en amo y esclavo de sí mismo. En Hiperculturalidad, y llevando este aspecto al ámbito digital, Han llega a hablar del eros de la conexión y cita a Heidegger para afirmar que hoy la creciente conectividad del mundo crea una abundancia y profusión de relaciones y posibilidades, pero que ese espacio de posibilidad saturado (del que cada vez nos resulta más difícil salir), reduce el proyecto vital y la libertad de elección a una mera posibilidad heredada (Han, 2018, 26-27); una suerte de erótica que no remite siquiera a otro. Los tiempos en los que existía el otro se han ido, dice al comienzo de La expulsión de lo distinto. "El otro como misterio, el otro como seducción, el otro como infierno, van desapareciendo» (Han, 2018, 9). Y como quiera que el signo patológico de los tiempos actuales no es tanto la represión cuanto la depresión, la presión destructiva no viene del otro sino del interior. Va a ser la misma necesidad de estar a la altura, de trabajar de forma omnipresente y constante, de consumirse y consumir, la que va a desarrollar unos rasgos autoagresivos. «El sujeto que, viéndose forzado a aportar rendimientos, se vuelve depresivo en cierta manera se muele a palos o se asfixia a sí mismo» (Han, 2018, 9-10).

Esta es, al fin, la clave del mapa que tratamos de describir: el protagonista, el sujeto sometido, no es del todo consciente de su sometimiento. $Y$ es también, como veremos, una de las razones morales últimas sobre las que se trata de fundamentar la ley de desconexión digital, a veces incluso de forma un tanto paternalista: el individuo a veces inconsciente, idiotizado en la masa, necesita la protección, al menos jurídica, por parte del Estado. Para explicar la situación en la que nos encontramos, en Psicopolítica, Han habla de un big brother amable, que no facilita el desvelamiento de las verdaderas intenciones del poder: en nuestra sociedad, "el consumo no se reprime, se maximiza. Se nos anima a comunicar y a consumir. El smartphone sustituye a la cámara de tortura. El big brother tiene un aspecto amable. La eficacia de su vigilancia reside en su amabilidad» (Han, 2018, 61).

Han es la voz actual más autorizada, pero no es la única. Jaron Lanier, el padre del término realidad virtual, lleva tiempo arremetiendo contra el peligro de que formemos parte de un rebaño digital. Lanier apunta contra lo que llama la cultura nerd o el maoísmo digital, es decir, la tendencia de la comunidad tecnológica de primar la forma (la plataforma) sobre el contenido y, consecuentemente, sobre las personas (Lanier, 2011). En su última obra, no se esconde. Desde el título lo deja bien claro cuando aporta diez razones para abandonar de inmediato nuestras redes sociales. Aunque no cita explícitamente ninguna razón vinculada al deterioro de la vida laboral, sus aportaciones, en este sentido, aparecen desde el principio del libro cuando afirma, por ejemplo, que somos animales de laboratorio, que los algoritmos se atiborran de datos sobre nosotros cada segundo, y que un mal uso de la tecnología nos está haciendo mucho daño, hasta el punto de modificar nuestra con- 
ducta y repercutir en todos los ámbitos de nuestra vida (Lanier, 2018, 17-25). En una línea similar, el psiquiatra alemán, Manfred Siptzer ha publicado recientemente un libro titulado Demencia digital, en el que va desgranando estudios para justificar afirmaciones como que los niños y los adolescentes pasan hoy más del doble del tiempo con medios digitales que en la escuela. Eso, cuando en la escuela no gira todo también en torno a las pantallas. Las consecuencias son, entre otras, trastornos del lenguaje y del aprendizaje, déficit de atención, estrés, depresiones y una disposición creciente a la violencia (Spitzer, 2013). Pero no es cosa solo de niños y adolescentes. Una de las consecuencias más demoledoras en la población adulta es la tendencia creciente a la multitarea, que acaba por trastornar la atención. $Y$ cita un reciente estudio norteamericano para mostrar que las personas de la sociedad digital interrumpimos nuestro trabajo cada once minutos de promedio y que las personas en las que predomina la multitarea son más superficiales en sus expresiones y relaciones, y más ineficaces en su trabajo (Spitzer, 2013, 223). Por último, diversos autores establecen la relación que existe entre esta sociedad digital, narcisista, del espectáculo y del rendimiento, y la salud mental. Especialmente interesantes son, a este respecto, las aportaciones de Sánchez-Carbonell y Beranuy que abordan la adicción a internet como sobreadaptación social y que, entre los grupos de riesgos, incluyen a las personas que atraviesan situaciones estresantes o con necesidades especiales (Talarn, 2007, 354); una perspectiva que profetiza Han cuando afirma que el hombre con sus aparatos digitales vive ya hoy la vida sin cosas de mañana. Nos dirigimos hacia un nuevo modelo de sociedad en el que el hombre del futuro ya no necesitará manos porque no tendrá que habérselas con la materia, tal y como la entendíamos hasta ahora. Un nuevo modelo también laboral que, al tiempo que lo convertirá todo en trabajo, rehuirá también de ese trabajo mismo. Puede parecer que nos dirigimos hacia un tiempo en el que el protagonista será más un homo ludens que un homo faber, pero la promesa esconde una trampa: el principio del rendimiento quita al juego todo lo lúdico y lo convierte de nuevo en trabajo. La diversión no es lo otro del trabajo, sino su producto, y los jugadores/trabajadores se convertirán en sujetos agotados, que duermen de la misma manera en que se duerme la pierna. Su relajación no es más que un modo de trabajo, en la medida en que sirve para la regeneración de la fuerza laboral. En resumen:

Hoy, en efecto, estamos libres de las máquinas de la era industrial, que nos esclavizaban y explotaban, pero los aparatos digitales traen una nueva coacción, una nueva esclavitud. Nos explotan de manera más eficiente por cuanto, en virtud de su movilidad, transforman todo lugar en un puesto de trabajo y todo tiempo en un tiempo de trabajo. La libertad de la movilidad se trueca en la coacción fatal de tener que trabajar en todas partes. En la época de las máquinas, el trabajo estaba ya delimitado frente al no-trabajo por la inmovilidad de las máquinas. El lugar de trabajo, al que había que desplazarse, se podía separar con claridad de los espacios de no trabajo. En la actualidad esta delimitación está suprimida por completo en muchas profesiones. El aparato digital hace móvil el trabajo mismo. Cada uno lleva consigo de aquí para allá el puesto de trabajo como un campamento. Ya no podemos escapar del trabajo (Han, 2013, 59). 


\subsection{La adicción digital: algunos datos sobre el estado de la cuestión}

¿Podemos afirmar que existe un tipo de dependencia a la que, con carácter general, denominamos adicción digital? ¿La reconocemos de la misma forma que lo hacemos con otras adicciones clásicas como pueden ser el tabaco, el alcohol o el juego? La primera respuesta es polémica, pero a mi juicio es afirmativa y la segunda es negativa. Volvemos a Han, que lo resume de esta forma en el prólogo de En el enjambre:

Ante el vertiginoso crecimiento del medio electrónico, Marshall McLuhan, teórico de los medios, advertía en 1964: la tecnología eléctrica está ya dentro de nuestros muros y estamos embotados, ciegos, sordos y mudos, ante su encuentro con la tecnología de Gutenberg. Algo semejante sucede hoy con el medio digital. Somos programados de nuevo a través de este medio reciente, sin que captemos por entero el cambio radical de paradigma. Cojeamos tras el medio digital, que, por debajo de la decisión consciente, cambia decisivamente nuestra conducta, nuestra percepción, nuestra sensación, nuestro pensamiento, nuestra convivencia. Nos embriagamos hoy con el medio digital, sin que podamos valorar por completo las consecuencias de esta embriaguez. Esta ceguera y la simultánea obnubilación constituyen la crisis actual (Han, 2014, 16).

Y desarrolla después una visión de los dispositivos móviles, que si bien, a mi juicio, se aparta del justo término medio virtuoso que propondré después, es sugerente como marco teórico para esta aproximación a la adicción digital:

El smartphone es un aparato digital que trabaja con un input-output pobre en complejidad. Borra toda forma de negatividad. Con ello se olvida de pensar de una manera compleja. Y deja atrofiar formas de conducta que exigen una amplitud temporal o una amplitud de mirada. Fomenta la visión a corto plazo, y ofusca la larga duración y lo lento. El me gusta sin lagunas engendra un espacio de positividad. La experiencia, como irrupción de lo otro, en virtud de su negatividad interrumpe el narcisismo imaginario. La positividad, que es inherente a lo digital, reduce la posibilidad de tal experiencia. La positividad continúa igual. El teléfono inteligente, como lo digital en general, debilita la capacidad de comportarse con lo general [...]. Los aparatos digitales traen una nueva coacción, una nueva esclavitud. Nos explotan de manera más eficiente por cuanto, en virtud de su movilidad, transforman todo lugar en puesto de trabajo y todo tiempo es un tiempo de trabajo. La libertad de la movilidad se trueca en la coacción fatal de tener que trabajar en todas partes [...] la comunicación digital hace que se erosione fuertemente la comunidad, el nosotros. Destruye el espacio público y agudiza el aislamiento del hombre (Han, 2014, 29). 
Sí, existe la adicción digital, seductora y esclavizante a un tiempo. Y empieza a existir, a mi juicio, un serio problema de salud pública. Madrid acaba de abrir el primer servicio de atención en adicciones tecnológicas. Ya no es solo una cuestión de unos cuantos teóricos que siempre ven calamidades y nubarrones en el horizonte. Se trata de un equipo de ocho profesionales, terapeutas que trabajan con los casos que les llegan, sobre todo, desde los servicios sociales de los ayuntamientos, centros de salud o centros escolares. Tiene el objetivo de ayudar de forma integral a las familias a hacer un buen uso de las nuevas tecnologías y de tratar a aquellas personas (está especialmente dirigido a adolescentes) que ya presenten un problema de adicción ${ }^{2}$.

Un estudio de profesores de la Universidad Estatal de San Diego, en California, afirma que el nivel de infelicidad se incrementa de forma constante en los adolescentes que pasan más de una hora diaria enganchados a la pantalla. Se ha observado que los usuarios de videojuegos, de entre 10 y 15 años de edad, que dedicaban al día más de tres horas a jugar en las pantallas, se sentían menos satisfechos con sus vidas y tienen más dificultades para gestionar sus emociones correctamente. Esos adolescentes pasan hoy una media de entre cinco a siete horas delante de las pantallas. En España, el Ministerio de Sanidad ha incluido por primera vez en 2018 las adicciones a las nuevas tecnologías en el Plan Nacional de Adicciones. Desde el organismo público se subraya que el principal grupo de riesgo son los adolescentes y los jóvenes y que el $18 \%$ de la población entre los 14 y los 18 años realiza un uso abusivo de las nuevas tecnologías. La Organización Mundial de la Salud no reconoce todavía la adicción a las nuevas tecnologías, aunque ya admite que existe la adicción a los videojuegos. Es un primer paso en un camino que, desgraciadamente, se ve venir. No es una cuestión baladí. Hay que abordarlo profesionalmente porque en estos jóvenes hiperexpuestos a la red, a menudo subyace un trastorno psicológico que se debe tratar cuanto antes mejor. De no atajarse a tiempo, de adultos pueden convertirse en personas inseguras, introvertidas, con miedo al rechazo y a la desaprobación social por su baja autoestima y obviamente con problemas asimismo en el ámbito laboral ${ }^{3}$.

Los especialistas sostienen que la terapia para dejar de hacerse un selfie diario es parecida a la de la drogadicción. Las adicciones del comportamiento (trabajo, juego, comida, compras, sexo, deporte, tecnología, etc.) responden a parámetros muy similares a los de las adicciones a las sustancias (alcohol, nicotina, heroína, cocaína, opiáceos, metanfetaminas, cafeína, esteroides, fármacos en general). Es decir, necesitan cada vez un consumo mayor para obtener satisfacción, generan agresividad en periodos de abstinencia, alteran los hábitos del sueño y de la alimentación, aíslan al sujeto hasta circunstancias extremas que le suponen un deterioro de sus relaciones sociales e inciden de manera muy negativa en la vida profesional (o en la educativa, en el caso de los menores). Los expertos hablan, a menudo, de que es necesario por lo menos un año para que ese hábito pernicioso se consolide como patología.

Tratando de sacar factor común, los especialistas hablan siempre de estas características a la hora de diagnosticar una adicción de este tipo:

\footnotetext{
2. Puede verse una referencia del citado centro en http://www.abc.es/espana/madrid/abci-madrid-abre-primer-servicio-para-adolescentes-adictos-movil-201804150103_noticia.html.

3. Para consultar al completo el estudio citado puede verse www.ikeepsafe.org/be-a-pro/balance/too-much-time-online: https://politica.elpais.com/politica/2018/03/02/actualidad/1520012219_058442.html

http://www.abc.es/familia/educacion/abci-terapia-para-dejar-hacerse-selfies-diarios-parecida-dejar-drogas-201708081753_noticia.html
} 
- Dependencia psicológica, que incluye el deseo, ansia o pulsión irresistible y la polarización de la atención, la modificación del estado de ánimo y la incapacidad de control.

- Efectos perjudiciales en el ámbito intrapersonal (experimentación subjetiva de malestar) e interpersonal (conflictos familiares, laborales, académicos, económicos, legales, etc.

- Síntomas de abstinencia tanto físicos como psicológicos (alteraciones de humor, irritabilidad, impaciencia, inquietud, tristeza, ansiedad, etc.).

- Negación, ocultación y/o minimización de lo que está sucediendo.

- Riesgo de recaída y de reinstauración de la adicción (en este sentido hay estudios todavía abiertos para observar si, como sucede en otras adicciones, el adicto corre el riesgo de recaer).

La aceptación e incluso el reconocimiento social que otorga la hiperconexión complica mucho el problema, porque, aunque también sucede en otras adicciones, en la adicción digital es muy frecuente pedir ayuda solo cuando la situación es extrema y ya se nos ha ido por completo de las manos.

Adam Alter, gurú actual sobre cuestiones de adicciones digitales, que ha arrasado con su provocadora propuesta, al preguntarnos a bocajarro « ¿quién nos ha convertido en yonquis tecnológicos?», es muy sugerente cuando se remonta a los usos primitivos de la palabra adicción. Ser adicto, en el mundo clásico, significaba tener una pasión. No solo no había nada malo en ser adictos sino que contaba con una cierta aceptación y reconocimiento sociales. Sin embargo, en el siglo xix, el campo de la medicina le otorgó el sentido con el que hoy conocemos el término. Fue la psicóloga norteamericana Kimberly Young, hoy directora del primer centro hospitalario del país para tratar las adicciones digitales que abrió en 2010. Parece claro que responde, sin duda, al auge de las nuevas tecnologías y, muy vinculadas a ellas, de las adicciones del comportamiento. Alter ha descrito la transición que se ha producido entre unas adicciones y otras cuando afirma que ya no son solo los químicos los que idean sustancias adictivas sino también los emprendedores los que diseñan experiencias que pueden atraparnos en comportamientos adictivos. De alguna manera, todos llevamos un adicto dentro. La razón principal las adicciones del comportamiento tienen un componente biológico. La palabra clave es dopamina. Las adicciones no se limitan al gusto; los adictos no son necesariamente individuos a los que les gustan las drogas que consumen, sino personas que ansían esas drogas con todas sus fuerzas, incluso cuando dejaban de gustarles porque estaban destruyendo sus vidas. Lo que hace que las adicciones sean tan difíciles de tratar es que cuesta mucho más luchar contra esa ansia que contra el gusto por la droga. En ese embrollo de gusto y ansia han encallado muchos estudios sobre adicciones. Y he aquí el quid de la cuestión. Alter tiene razón cuando afirma, poniendo resultados científicos encima de la mesa, que a pesar de que gustar y querer suelen ir de la mano, cuando se trata de adicciones cada una sigue su camino.

Igual que las drogas desencadenan la producción de dopamina, las señales comportamentales también lo hacen. Cuando un adicto a los videojuegos enciende el portátil, sus niveles de dopamina se disparan; cuando una adicta al ejercicio se ata las zapatillas de correr, sus niveles de dopamina también se disparan. A partir de este momento, estos adictos del com- 
portamiento se parecen mucho a los adictos a las drogas. Las adicciones no están motivadas por las drogas o los comportamientos en sí, sino por la idea aprendida con el tiempo, de que son capaces de proteger a los adictos de su malestar psicológico (Alter, 2018, 80-81).

En realidad, Alter nos quiere hacer pensar sobre cómo en muchas ocasiones las intuiciones que tenemos sobre las adicciones no se corresponden en absoluto con la realidad. No es, por ejemplo, que el cuerpo se enamore de pronto de una droga sin ser correspondido, sino que nuestra mente aprende a asociar la sustancia o el comportamiento en cuestión con el alivio del sufrimiento psicológico. Y resulta que en el nuevo continente digital esas asociaciones se producen con una alta frecuencia, entre otras cosas porque lo digital nos facilita la consecución de numerosos pequeños objetivos que andamos persiguiendo a diario, intentado culminar unos sin haber conseguido otros. Esta multitarea, como hemos visto, es un caldo de cultivo para la adicción.

Si se hace con moderación, establecer objetivos personales cumple una función intuitiva, porque te dice cómo invertir tu limitada cantidad de tiempo y energía. Pero hoy día los objetivos llaman a nuestra puerta sin que los hayamos invitado. Si te unes a una red social, no tardarás en querer atraer más seguidores y likes. Si te creas una cuenta de correo electrónico, siempre estarás pendiente de mantener la bandeja de entrada vacía. Si juegas al Candy Crush, necesitarás batir tu propio record [...]. Estos objetivos se acumulan y alimentan actividades adictivas que llevan al fracaso o, quizá peor aún, a una sucesión de éxitos que engendrará un objetivo ambicioso tras otro (Alter, 2018, 105-106).

A esto le tenemos que añadir que, en el caso de los entornos digitales, el feedback que todos buscamos en nuestros procesos de aprendizaje se producen de forma inmediata. Se puede tener casi todo y se puede tener casi al instante. En ese casi reside el gancho tramposo con el que toda adicción nos atrapa. Nuestros objetivos en la red se irán acumulando al tiempo que se acumulan actividades adictivas que no nos llevan directamente al fracaso, sino casi al éxito. Vamos acumulando pequeños fracasos que percibimos como casi triunfos o, peor aún, triunfos que nos van a abrir una ventana tras otra, de manera hipertextual, al más puro estilo Windows y generando un objetivo ambicioso tras otro. Es, en definitiva, el modelo clásico de las máquinas tragaperras, minuciosamente calculado y diseñado para generar un comportamiento adictivo. No son mundos ajenos ni tan distantes. Parte del drama de la adicción digital es que, además de ser una adicción en sí misma, auxilia y es decisiva en el desarrollo de otras adicciones, sobre todo conductuales. Teletrabajamos, respondemos correos electrónicos las 24 horas del día; telecompramos, como usuarios premium que a las dos horas tienen lo que han pedido en casa; teleapostamos de forma ubicua y constante mientras vemos un partido de fútbol.

No sería honrado concluir el epígrafe dedicado a la adicción digital sin mencionar al menos que hay quienes tratan de negarla con argumentos razonables, aunque a mi parecer equivocados. Con la prudencia que se debe tener en los albores de una revolución de este calibre, como es la digital, hubo quienes a mediados de los años 90 del siglo pasado entendieron que 
la preocupación apocalíptica por la adicción a internet no era sino una percepción cultural y una especie de adicción al concepto de adicción (Suler, 1996). Erraban el tiro porque, en primer lugar, no se trata tanto de una preocupación apocalíptica, que en términos conocidos de Eco vendría a ser una profecía de calamidades, motivada por el planteamiento tecnófobo de quienes la defendían (Eco, 1964). Se trata de reconocer las grandes oportunidades que nos ofrece el continente digital, sopesando equilibradamente los riesgos y desafíos que conlleva.

Otros, más recientemente, han seguido defendiendo, contra las evidencias de los últimos estudios existentes que hablar de adicción a internet es una broma que ha sido tomada en serio. Entre los argumentos más interesantes están los que desglosa Eparquio Delgado en Los nativos digitales no existen. Delgado va describiendo los elementos necesarios para una adicción, de manera muy similar a como los hemos visto aquí, y va concluyendo uno a uno que no se cumplen los requisitos (sustancia o comportamiento y sus efectos biológicos, disposiciones psicológicas y análisis del contexto en el que se produce). Comienza argumentando que las clasificaciones diagnósticas de los organismos mundiales más relevantes del campo de la salud no han incluido la adicción a internet. Eso no quiere decir que no lo vayan a hacer en el futuro e incluso que no lo estén valorando ya en este momento (Lluna, S. y Pedreira, J., 2017).

Este punto ha cambiado notablemente en el poco más de un año transcurrido desde la publicación del mencionado estudio que niega la adicción. Hemos visto que en España ya se han dado los primeros pasos con organismos públicos y una preocupación expresa por parte del Gobierno. Los datos de las investigaciones más recientes apuntan, en efecto, hacia una presencia creciente del alto consumo digital en edades tempranas. El último estudio de la Asociación para la Investigación de Medios de Comunicación Social (AIMC) destaca que, en España, actualmente, la población infantil pasa casi cinco horas diarias frente a alguna pantaIla (236 minutos de media, de lunes a viernes; 396, los fines de semana). El tiempo de exposición aumenta conforme los niños van siendo más mayores. Entre 12 y 13 años permanecen una media de siete horas diarias ante las pantallas. El estudio pone de manifiesto también un descenso significativo en el consumo de todos los dispositivos clásicos. Solo el smartphone aumenta de manera relevante 4 . Nada parece indicar que, a corto plazo, la tendencia vaya a cambiar. Es aventurado predecir exactamente qué sucederá con esta generación, que en una década se incorporará al mercado laboral, pero sí parece claro que buena parte de su éxito personal y laboral dependerá de la manera en que sepan integrar con equilibrio las enormes ventajas que nos aporta el mundo conectado. Ante esta tesitura, no parece lo más indicado pasar mucho más tiempo debatiendo si la reconocemos y si llamamos o no por su nombre a la adicción digital.

Para Delgado «considerar que existe esta adicción no es inocuo porque, si aceptamos que el uso de internet en sí mismo puede ser peligroso por su potencial adictivo, había que tratarlo igual que hacemos con la cocaína o los ansiolíticos. Si el uso de internet o del móvil -añade- pudiera derivar en una adicción, podría considerarse su restricción legal a determinadas edades, o la adicción podría ser causa de baja laboral» (Lluna, S. y Pedreira, J., 2017, 77-78). En efecto, hacia ahí caminamos. Estamos viendo que hay incluso gobiernos que se han planteado la prohibición en determinados lugares y para determinadas edades. En

4. Estudio completo en www.aimc.es. 
Finlandia, por ejemplo, la adicción a internet en los jóvenes puede ser eximente del servicio militar obligatorio. Añade, no obstante, Delgado que se podría plantear que llamarlo adicción no supone ningún problema si eso nos sirve para visibilizar el problema y ofrecer soluciones. De eso se trata, exactamente. Por un lado, se trata de no caer en las redes de lo que Han definía como big brother amable y visibilizar el problema que ya tenemos entre nosotros. Y, por otro, de ofrecer soluciones, que en nuestro caso, pasan por pensar y repensar la ciencia, entendiendo que la teoría es ya un modo práctico de estar en el mundo, imprescindible para que acción y contemplación se integren adecuadamente.

\section{El derecho a la desconexión digital}

\subsection{Repensar la ciencia jurídica desde la razón abierta: el diálogo con la ética y los fundamentos prejurídicos}

Se ha podido intuir ya, pero, antes de adentrarnos en las claves de la legislación que viene sobre el derecho a la desconexión digital, es conveniente explicitar que apostamos en el enfoque metodológico por un repensamiento de las ciencias. Lo hacemos desde el concepto ratzingeriano de razón abierta. Ante una cultura positivista y relativista, Joseph Ratzinger (posteriormente, Benedicto XVI) hizo notar la profunda división de saberes y la excesiva especialización, particularmente en el ámbito universitario, que a su juicio se salva con un ensanchamiento de la razón, es decir, con una visión amplia y abierta de la razón y de su ejercicio en la apasionante e inagotable tarea de la búsqueda de la verdad y de la respuesta a las preguntas fundamentales sobre el hombre y su destino. El concepto de razón tiene así que ensancharse para ser capaz de abarcar y explorar los aspectos de la realidad que van más allá de lo puramente empírico y lograr una síntesis armoniosa de saberes que integran la teología y la filosofía para poder comprender la realidad respetando su realidad metafísica. En su discurso para La Sapienza, que lamentablemente no pudo ser pronunciado, Benedicto XVI, lo escribió así:

Hoy, el peligro del mundo occidental-por hablar solo de este-es que el hombre, precisamente teniendo en cuenta la grandeza de su saber y de su poder, se rinda ante la cuestión de la verdad. Y eso significa al mismo tiempo que la razón, al final, se doblega ante la presión de los intereses y ante el atractivo de la utilidad, y se ve forzada a reconocerla como criterio último. Dicho desde el punto de vista de la estructura de la universidad: existe el peligro de que la filosofía, al no sentirse ya capaz de cumplir su verdadera tarea, degenere en positivismo; que la teología, con su mensaje dirigido a la razón, quede confinada a la esfera privada de un grupo más o menos grande. Sin embargo, si la razón, celosa de su presunta pureza, se hace sorda al gran mensaje que le viene de la fe cristiana y de su sabiduría, se seca como un árbol cuyas raíces no reciben ya las aguas que le dan vida. Pierde la valentía por la verdad y así no se hace más grande, sino más pequeña. Eso, aplicado a nuestra cultura europea, significa: si quiere solo 
construirse a sí misma sobre la base del círculo de sus propias argumentaciones y de lo que en el momento la convence, $y$, preocupada por su laicidad, se aleja de las raíces de las que vive, entonces ya no se hace más razonable y más pura, sino que se descompone y se fragmenta 5 .

En el ámbito jurídico se percibe de forma clara el riesgo del mencionado positivismo. En sus diferentes variantes (excluyente, incluyente y axiológico), el positivismo jurídico es incapaz de servir como herramienta para dar cuenta de la realidad. Su pretensión de dar a la teoría del Derecho un carácter meramente descriptivo, implica la exclusión de la dimensión valorativa de las normas jurídicas (Atienza y Manero, 2007).

¿Cómo prescindir de cualquier consideración moral en la formulación, por ejemplo, del delito de omisión de socorro, que en el artículo 195.1 del Título IX de la Ley Orgánica 10/1995, de 23 de noviembre, del Código Penal, explicita la pena que se nos impondrá si no socorriéramos a una persona que se hallara desamparada y en peligro manifiesto y grave, cuando pudiéramos hacerlo sin riesgo propio ni de terceros? ¿No es fácil atisbar aquí una suerte de humanismo del rostro, que, en palabras de Lévinas, nos señale la verdadera manera de relacionarnos con los demás, que incluye una dimensión ética, que me vincula y me requiere imperativamente? (Levinás, 1997) ¿Cómo no abrir la ciencia jurídica a dialogar con la filosofía (aquí lo haremos específicamente con la ética) o con la misma teología? Sí, en efecto, estamos hablando de ese enemigo del derecho, viviente y positivo, que no es otro que el derecho natural. Dicho derecho ha sido entendido con frecuencia, desde un punto de vista general, con referencia absorbente a la palabra naturaleza, comprendida como las vicisitudes y alternativas históricas, que ha dado lugar a su vez a una explicación abstracta y esencialmente racional, o mejor racionalista, del mundo jurídico. Aquí los filósofos, en gran medida, han despreciado el derecho natural como un grotesco sustitutivo del derecho teórico (espíritu y método del derecho natural cristiano), porque el término se ha entendido como típicamente expresivo de una corriente de pensamiento: el iusnaturalismo, pero el derecho natural no es solamente el racionalista, individualista y antihistórico del iusnaturalismo. Al lado y por encima del iusnaturalismo propiamente dicho, se sitúa la doctrina del derecho natural inscrita en la tradición escolástica de la llamada philosophia perennis, originada en la antigüedad oriental y grecorromana, y que pasa a través del hecho único de la revelación cristiana y llega a san Agustín o santo Tomás. La idea de derecho natural presupone así un concepto de naturaleza en que naturaleza y razón se compenetran y complementan y en el que la naturaleza misma se torna racional.

En esa gran estela, podemos hablar, como lo hacen Ratzinger y Habermas, de fundamentos prepolíticos (y por analogía, de fundamentos prejurídicos) del Estado de derecho.

5. A pesar de que en ella habían estado Pablo VI, en 1964, y Juan Pablo II, en 1991, Benedicto XVI no pudo pronunciar la conferencia prevista en la Universidad de La Sapienza para el 17 de enero de 2008. El Cardenal Secretario de Estado vaticano se refería así por carta al Rector: «Lamentablemente, al faltar las condiciones para una acogida digna y tranquila del Papa, debido a las acciones de un grupo indudablemente minoritario de profesores y de alumnos, se ha juzgado conveniente diferir la visita programada para no dar motivos a manifestaciones que serían incómodas para todos". La Santa Sede hizo público el texto de la conferencia que debería haber pronunciado, en la que el Papa Ratzinger ahonda en las cuestiones tratadas previamente en la Universidad de Ratisbona sobre la relación entre fe y razón. Aquí puede leerse el discurso completo: http://w2.vatican.va/content/benedict-xvi/es/speeches/2008/january/documents/hf_benxvi_spe_20080117_la-sapienza.html 
En su diálogo sobre razón y religión, Ratzinger afirma que lo que cohesiona al mundo son los fundamentos morales y prepolíticos del Estado liberal (Habermas y Raztinger, 2008). En estrecha sintonía, hagámoslo, pues. Veamos, primero, cuáles son las claves principales de la legislación que viene sobre desconexión digital y fundamentemos después, desde la ética clásica, los cimientos que la sostienen.

\subsection{Las claves de la legislación que viene}

Con fecha 8 de octubre de 2018, el Boletín Oficial de las Cortes Generales publicó el informe emitido por la ponencia sobre el Proyecto de Ley Orgánica de Protección de Datos de Carácter Personal y Garantía de los Derechos Digitales (anteriormente llamada solo de Protección de Datos de Carácter Personal). Tras la habitual exposición de motivos, de la posteriormente rescataremos también algún fundamento ético, el proyecto se estructura en diez títulos, con un total provisional de 97 artículos, más 22 disposiciones adicionales, 6 transitorias, 1 derogatoria y 16 finales. A nosotros nos interesa especialmente, además de la exposición de motivos, el título décimo, que es totalmente nuevo, y que recoge una serie de derechos digitales que, a partir de ahora, deberán ser protegidos. Tan solo nueve días después, el 17 de octubre se publica el dictamen de la comisión ${ }^{6}$.

En primer lugar, el epígrafe IV de la exposición de motivos recoge lo siguiente:

Internet, por otra parte, se ha convertido en una realidad omnipresente tanto en nuestra vida personal como colectiva. Una gran parte de nuestra actividad profesional, económica y privada se desarrolla en la Red y adquiere una importancia fundamental tanto para la comunicación humana como para el desarrollo de nuestra vida en sociedad. Ya en los años noventa, y conscientes del impacto que iba a producir Internet en nuestras vidas, los pioneros de la Red propusieron elaborar una Declaración de los Derechos del Hombre y del Ciudadano en Internet. Hoy identificamos con bastante claridad los riesgos y oportunidades que el mundo de las redes ofrece a la ciudadanía. Corresponde a los poderes públicos impulsar políticas que hagan efectivos los derechos de la ciudadanía en Internet promoviendo la igualdad de los ciudadanos y de los grupos en los que se integran para hacer posible el pleno ejercicio de los derechos fundamentales en la realidad digital. La transformación digital de nuestra sociedad es ya una realidad en nuestro desarrollo presente y futuro tanto a nivel social como económico. En este contexto, países de nuestro entorno ya han aprobado normativa que refuerza los derechos digitales de la ciudadanía. Los constituyentes de 1978 ya intuyeron el enorme impacto que los avances tecnológicos provocarían en nuestra sociedad y, en particular, en el disfrute de los derechos fundamentales. Una deseable futura reforma de la Constitución debería

6. Puede consultarse el Proyecto de Ley en http://www.congreso.es/public_oficiales/L12/CONG/BOCG/A/BOCG-12-A-13-4. PDF. 
incluir entre sus prioridades la actualización de la Constitución a la era digital y, específicamente, elevar a rango constitucional una nueva generación de derechos digitales. Pero, en tanto no se acometa este reto, el legislador debe abordar el reconocimiento de un sistema de garantía de los derechos digitales que, inequívocamente, encuentra su anclaje en el mandato impuesto por el apartado cuarto del artículo 18 de la Constitución Española y que, en algunos casos, ya han sido perfilados por la jurisprudencia ordinaria, constitucional y europea.

Destacamos como aspectos clave los siguientes:

- La omnipresencia de la red, en el sentido de hiperconexión y sociedad de las pantallas que hemos tratado en el capítulo primero de este artículo.

- La incidencia fundamental en la vida humana, como oportunidad y también como riesgo.

- El reconocimiento de una serie de derechos digitales que, si bien hasta ahora no se contemplaban, ya se intuían en el apartado cuarto del artículo 18 de la Constitución Española, donde se hace referencia a la necesaria limitación por ley del uso de la informática para garantizar el honor y la intimidad personal y familiar de los ciudadanos, y que asimismo ya han sido perfilados en diferentes jurisprudencias.

Por otra parte, los mencionados derechos «de nueva creación» que aparecen en el Título X son: derecho de acceso universal a internet, a la neutralidad en la red, a la seguridad digital, a la educación digital, a la protección de los menores en Internet, de rectificación en Internet, a la actualización de informaciones en medios de comunicación digitales, a la intimidad y uso de dispositivos digitales en el ámbito laboral, a la intimidad frente al uso de dispositivos de videovigilancia y de grabación de sonidos en el lugar de trabajo, a la intimidad ante la utilización de sistemas de geolocalización en el ámbito laboral, derechos digitales en la negociación colectiva, al olvido en búsquedas de internet, en servicios de redes sociales y equivalentes, a la portabilidad en servicios de redes y equivalentes, derecho al testamento digital, y muy particularmente, en lo que a nosotros respecta, derecho a la desconexión digital en el ámbito laboral (artículo 88), que dice así:

1. Los trabajadores y los empleados públicos tendrán derecho a la desconexión digital a fin de garantizar, fuera del tiempo de trabajo legal o convencionalmente establecido, el respeto de su tiempo de descanso, permisos y vacaciones, así como de su intimidad personal y familiar.

2. Las modalidades de ejercicio de este derecho atenderán a la naturaleza y objeto de la relación laboral, potenciarán el derecho a la conciliación de la actividad laboral y la vida personal y familiar y se sujetarán a lo establecido en la negociación colectiva o, en su defecto, a lo acordado entre la empresa y los representantes de los trabajadores.

3. El empleador, previa audiencia de los representantes de los trabajadores, elaborará una política interna dirigida a trabajadores, incluidos los que ocupen puestos directivos, en la que definirán las modalidades de ejercicio del derecho a la desconexión y las acciones de formación y de sensibilización del personal sobre un uso razonable de 
las herramientas tecnológicas que evite el riesgo de fatiga informática. En particular, se preservará el derecho a la desconexión digital en los supuestos de realización total o parcial del trabajo a distancia así como en el domicilio del empleado vinculado al uso con fines laborales de herramientas tecnológicas.

Aquí van también tres aspectos clave, antes de proponer los fundamentos éticos que se derivan de cuanto hemos dicho:

- Reconocimiento de la separación de ámbitos de construcción personal (vida íntima, privada, pública) como algo bueno y deseable para el trabajador.

- Reconocimiento asimismo de la bondad de la conciliación entre ámbitos, especialmente entre el familiar (que se desenvuelve mayormente en las esferas privada e íntima) y el labora (que transcurre sobre todo en las esferas privada y pública).

- Uso razonable (equilibrio y virtud) y alarma ante la fatiga (sociedad del cansancio descrita por Byung Chul-Han).

Concluimos poniendo en relación ética y derecho para, en la línea de lo explicitado anteriormente, desvelar los fundamentos prejurídicos de la norma. Lo hacemos con cinco fundamentos de la ética clásica, aquella que nace en la Antigüedad, como reflexión acerca de los contenidos de la vida buena y que conserva grosso modo estas señas de identidad a lo largo de veinte siglos, hasta finales del siglo xvIII cuando experimenta un cambio de rumbo y, en concreto, una restricción de sus intereses, pasando de ser una teoría de la vida buena a ser casi exclusivamente una teoría del deber (Rodríguez Duplá, 2006). Así, con raíces socráticas, platónicas y aristotélicas, fundamentamos la necesidad del cuidado de uno mismo, de aspirar a una vida lograda, de distanciarnos prudencialmente de la mera producción que caracteriza al homo faber y de una recuperación de la virtud y el término medio aristotélicos. Todos ellos, en diferente medida, son sustento del derecho a la desconexión digital que tenemos a la vuelta de la esquina.

\subsection{Cuatro fundamentos desde la ética clásica}

\subsubsection{El cuidado del alma o el cuidado de sí}

Conocemos el relato: para castigarle por su vanidad, Némesis, la diosa de la venganza, hizo que el joven y apuesto Narciso se enamorara de su propia imagen reflejada en una fuente. El mito anticipa de forma preclara la cultura del selfie: Narciso, embebido de su yo reflejado, e incapaz de apartar la mirada de su imagen, acaba por arrojarse a las aguas. La narración simbólica no puede encarnar mejor ese aspecto sombrío de la condición humana que, en el tiempo que nos ha tocado vivir, se manifiesta como una feroz amalgama de omnipresencia en las redes, envanecimiento e imprudencia. En La resistencia íntima, Josep María Esquirol, propone con lucidez reinventar la mirada y recuperar la pausa, la proximidad, el silencio y la reflexión ante la inmediatez compulsiva y ese estado de permanente exposición pública que nos asola y que nos arroja a la monocromía de un mundo en exceso tecnificado (Esquirol, 2015). $Y$ es en ese territorio minado donde aborda sin piedad a los narcisos que, por diver- 
sos motivos, confundieron lo que la tradición socrática llama cuidado del alma o cuidado de sí, con una suerte de vigorexia existencial, que alcanza su paradigma en la obsesión por el cuerpo que se machaca en los gimnasios. No se trata del equilibrio necesario para propiciar el conocido mens sana in corpore sano, sino un rebasamiento del cuerpo mismo, que en su cuidado extremo llega a manifestar diferentes enfermedades contemporáneas, entre ellas, la que el Papa Francisco llama con ácida gracia la enfermedad del espejo. Ante la necesaria pausa, que cuida de uno e invita al mismo tiempo a salir de uno mismo, el narcisismo irrumpe como seña de identidad de la cultura contemporánea, en un replegarse del yo sobre sí mismo que trata de prescindir de todo vínculo.

Sin embargo ese cuidado de sí, esa pausa de asombro y contemplación primeras, son imprescindibles hasta el punto de estar vinculadas a la salud integral del ser humano. En la tabla de competencias digitales docentes del Ministerio aparecen las relacionadas con el uso equilibrado de la tecnologías, como amenazas para la integridad física y el bienestar psicológico (especialmente la adicción digital), dentro del apartado 4.3 dedicado a la «protección de la salud", que a su vez forma parte del área competencial número 4, que es la dedicada a la seguridad. El artículo 8.3 del Proyecto de Ley se refiere indirectamente también a esto cuando habla de la obligación del empleador de elaborar una política interna de sensibilización del personal sobre un uso razonable de la tecnología que evite el riesgo de fatiga informática. El sociólogo y antropólogo francés David Le Breton retrata el envés de ese cuidado de sí al dibujar la desaparición de sí como una tentación contemporánea (Le Breton, 2016). El placer de vivir que ansiamos no es fácil de encontrar, menos aún en una sociedad que exige estar constantemente a la altura y en la que el individuo está en permanente construcción. Muchos de nuestros contemporáneos buscan aliviar la presión que se vuelve insoportable sobre sus espaldas, desapareciendo, suspendiendo su existencia, dándose tregua por un instante. La adicción digital sería una de esas formas de desaparición, paradójicamente necesarias, pero peligrosas al mismo tiempo para el aludido cuidado de sí. En cualquier caso, el fundamento socrático no deja lugar a la duda. El final del Gorgias es esclarecedor cuando afirma que va a todas partes sin hacer otra cosa que intentar persuadir, a jóvenes y viejos, a no ocuparse de los cuerpos ni de los bienes antes que del alma, con tanto afán, a fin de que ésta sea lo mejor posible. En versión Ikea, darse a la conversación, por otra parte eminentemente socrática; desconectarse de lo digital para cuidarse y poder reconectar después con mayor brío.

\subsubsection{La vida mejor, fuera de la caverna}

Represéntate hombres en una morada subterránea en forma de caverna, que tiene la entrada abierta, en toda su extensión, a la luz. En ella están desde niños con las piernas y el cuello encadenados, de modo que deben permanecer allí y mirar solo delante de ellos, porque las cadenas les impiden girar en derredor la cabeza.

La alegoría de Platón es, en su comienzo, perturbadora. Nos muestra la situación en la que se halla la naturaleza humana, es decir, el estado en el que se encuentran la mayoría de los hombres con respecto al conocimiento de la verdad o la ignorancia. En la caverna, encadenados, no pueden siquiera girar la cabeza. No es de extrañar que, cuando es liberado 
un prisionero, la luz le llene los ojos de fulgores y que necesite tiempo para acostumbrarse, para poder llegar a mirar las cosas de arriba.

La imagen simbólica de unos hombres atados de piernas y cuello, desde niños, mirando al frente, tiene su correlato posmoderno en los hikikomoris, una palabra japonesa que nos sirve para nombrar a los muchachos que en nuestros días sufren un aislamiento social agudo y que huyen de su incapacidad para las relaciones sociales buscando refugio en otras vidas. El término hikikomori fue acuñado por el psiquiatra Tamaki Saito, y significa literalmente estar recluido, apartarse, pero a diferencia de otros, este apartarse, este retiro exacerbado, no tiene nada que ver con la vida lograda. Saito la definió como una enfermedad social, basada en la reclusión intencional, durante al menos un periodo de seis meses (Saito, 2013). La soledad del hikikomori es la del que quiere, pero no puede abrazar la vida, la del que tiene dominada la voluntad, la del que experimenta, en terminología del profesor López Quintás, más vértigo que éxtasis. Los procesos de éxtasis nos ofrecen posibilidades de crecimiento personal, y requieren desprendimiento y generosidad; los de vértigo, sin embargo, son fruto de la cerrazón sobre nosotros mismos, acaban por bloquear nuestro desarrollo y anularnos la personalidad. El proceso de vértigo, como fácilmente acertamos a descubrir en la hiperconexión, es falaz y traidor, porque nos promete al principio una vida intensa y nos lanza por una pendiente de excitaciones crecientes que, en realidad, no hacen sino apegarnos al mundo de lo material, de las sensaciones que con ello experimentamos, y nos aleja de la vida creativa. Las ideas fluyen cuando dejamos reposar a la mente, cuando tenemos espacio y tiempo sosegados para poner conceptos y experiencias en relación. $Y$ se aturden cuando nos ocupamos, en modo multitarea, a todo aquello que nos va surgiendo entre pantalla y pantalla. El vértigo nos aleja del encuentro, nos ciega para los grandes valores, nos somete a todo tipo de riesgos y, en consecuencia, disminuye al máximo nuestra capacidad de unirnos a las realidades del entorno (López Quintás, 2006). Cuando no somos capaces de mirar más allá del ombligo de nuestros dispositivos móviles, hasta incluso el extremo patológico que nos ocupa, nos sumimos en una espiral de egoísmo, goce, euforia, exaltación superficial, decepción, tristeza, angustia, soledad asfixiante y destrucción, como secuencia de una experiencia vertiginosa que conocen bien quienes se encierran en su habitación.

Hay sociólogos que afirman que Japón ha desarrollado el caldo de cultivo necesario para que haya tenido lugar allí un crecimiento tan rápido del fenómeno. La explicación más plausible sería la de la brusca adaptación que la cultura nipona ha tenido que llevar a cabo en muchos ámbitos, con particular incidencia en lo que se refiere a la integración de Japón en un mercado laboral occidentalizado. Pero nunca un fenómeno complejo puede explicarse por una sola causa. El desplome de su sistema educativo (referente internacional hace apenas dos décadas), la deficiente educación emocional de los jóvenes-adultos, que por supuesto no se da solo en Japón, pero que ha hecho que muchos no supieran gestionar adecuadamente sus frustraciones, y su peculiar relación con la tecnología son factores que no debemos olvidar a la hora de plantear un análisis sobre el aislamiento social agudo de tantos japoneses. Es cierto que si comparamos los dos millones de afectados en Japón con unos pocos centenares en España, puede parecernos poca cosa, pero es muy significativo que en los últimos años hayan empezado a conocerse cifras y los nombres de los primeros especialistas que en nuestro país han abordado el problema profesionalmente. En noviembre de 2014, médicos del Instituto de Neuropsiquiatría y Adicciones del Hospital del Mar, de Barcelona, 
presentaron el primer estudio europeo sobre las características clínicas y sociodemográficas del síndrome de hikikomori (Malagón-Amor et al., 2015). Se evaluaron 164 casos de toda España, la mayoría de los analizados llevaban con un trastorno mental asociado, aunque ya se apuntaban las dificultades para identificar los casos, dado que en muchas ocasiones no se podía llegar a pacientes que no presentaran otra patología asociada al aislamiento social. En aquel estudio pionero, el periodo medio de aislamiento se situaba en 39,3 meses, con un caso extremo investigado de 30 años de abandono. El perfil era distinto al japonés, porque, aunque predominaban los varones, la edad media era de 36 años.

Para muchos hikikomoris, entregados a la espiral del vértigo, la vida es una fuente de angustia. Hay que huir de ella y del absurdo que nos tiene como a Sísifo empujando una enorme roca montaña arriba, condenados a repetir una y otra vez la tarea porque la roca rueda ladera abajo al llegar a la cima. ¿Qué cabe si no pensar, con Sartre, que en esta tesitura el infierno son los otros? ¿Qué cabe si no refugiarse porque el otro es un usurpador que ha entrado en mi entorno y me ha echado del lugar privilegiado que yo ocupaba? Pues cabe, afortunadamente, entender que el otro me mejora, me interpela, me pide no cosificarle, desvirtualizarle, y salir cuanto antes de la habitación, porque quien llama con los nudillos a la puerta es siempre un quién, nunca un qué, y, por desgracia, esto que alcanzamos a ver con tanta claridad en el abrazo no siempre se manifiesta con tal nitidez en la webcam.

Para no llegar al extremo hikikomori, en el artículo 88.1 la legislación apunta la necesidad de respetar los tiempos de descanso y vacaciones, y la mencionada tabla de competencias digitales que apunta en reiteradas ocasiones el riesgo de adicción digital, como cuando en el nivel básico de la protección de la salud se refiere a conocer que un mal uso de la tecnología puede afectar negativamente en la salud, en el nivel intermedio cuando se refiere a entender que entre esos riesgos para la salud están desde los aspectos ergonómicos hasta la adicción a las tecnologías, o cuando en el nivel avanzado menciona la necesidad de compartir en comunidades educativas hábitos saludables de uso de la tecnología, fomentándolos y compartiéndolos especialmente en redes sociales.

El platónico mito de la caverna nos enseña al menos dos cuestiones esenciales para nuestra saludable desconexión:

- La realidad se nos da a menudo por representaciones, pero no todo es representación.

- Hay cavernas oscurísimas en el mundo (basta con ver el ejemplo de los hikikomoris para darse cuenta), pero no todo el mundo es una caverna y tenemos derecho a desconectar, aunque solo sea para que, cuanto antes, deje de deslumbrarnos la luz del sol y podamos acostumbrarnos a ella.

\subsubsection{Homo faber $y$ vita contemplativa}

El artículo 88.2 del Proyecto de Ley se basa en la necesidad de preservar la conciliación laboral/ familiar. En La vida lograda, Alejandro Llano sostiene que una de las causas principales de la convicción moderna de que el poder político va unido irremediablemente a la corrupción surge de la interna escisión que la misma ética ha sufrido, «al aceptarse como inevitable -incluso como deseable- la separación entre moral pública y moral privada, como si la casa y el ágora fuera ámbitos en los que rigen planteamientos éticos diversos» (Llano, 2002, 104). 
García Morente en el elogio que hace de la vida privada sostiene que el estilo de vida no admite compartimentos estancos. La vida lograda comprende ambas dimensiones: la privada y la pública (Morente, 2011). La plenitud vital afectará inevitablemente a ambas esferas y el desequilibrio, la falta de conciliación, equivaldría a decir que una persona está sana por fuera y enferma por dentro, cuando entendemos con facilidad que si está sana (o si está enferma), lo estará a todos los efectos.

El error de separar la ética pública de la privada surge de situar la clave de la moral en las normas de la conducta en lugar de hacerlo en las virtudes. Dicho de otra forma, surge de situar el centro de nuestras preocupaciones más en el hacer que en el contemplar, o mejor dicho, en un hacer que no incluya una actividad contemplativa, que es la que debe nutrir y dar sentido a la acción. Sin contemplación se erosionan privacidad e intimidad. Y sin intimidad cultivada, todo en nosotros, en nuestras actuales circunstancias, corre el riesgo de convertirse en extimidad, es decir, en aquello que siendo muy íntimo y familiar se convierte a la vez en algo radicalmente extraño. Una paradoja que define al sujeto de nuestro tiempo, siempre exiliado de sí mismo, hasta el punto de que solo parece encontrar su ser más íntimo en lo más lejano y localizado de él (Miller, 2010). Han lo describe con tanta claridad que parece estar fundamentando, desde el punto de vista ético, la urgencia de una legislación en favor de la desconexión digital:

El animal laborans tardomoderno es, en este sentido estricto, todo menos animalizado. Es hiperactivo e hiperneurótico [...]. Durante la modernidad tardía todas las actividades humanas se han reducido al nivel del trabajo [...]. La pérdida de capacidad contemplativa, que está vinculada a la absolutización de la vida activa, es corresponsable de la histeria y el nerviosismo de la moderna sociedad activa (Han, 2012, 45-51).

\subsubsection{La virtud, el vicio y el término medio}

El presupuesto básico de la legislación que se está elaborando sobre desconexión digital se asienta en la bondad del equilibrio, del uso moderado de la tecnología, y en la prevención del abuso y la adicción tecnologías. Un uso razonable dice el artículo 88.3 del Proyecto de Ley. Aristóteles nos lo dijo en Ética a Nicómaco con la conocida sentencia acerca de la virtud que se hallaría en el término medio. La virtud es un hábito selectivo, que consiste en una posición intermedia para nosotros, determinada por la razón y tal y como la determinaría el hombre prudente. Posición intermedia entre dos vicios, el uno por exceso y el otro por defecto. Hábito, que contra la extendida creencia que prolifera en determinados manuales de autoayuda, no se traduce de forma cuantitativa (no son 21 días consecutivos los que se necesitan para acostumbrarse al gimnasio o para aprender inglés), sino que se trata más bien de una cuestión cualitativa, que tiene que ver con el mencionado dominio de la voluntad. ¿Queremos el bien, lo reconocemos, pero aun así no podemos hacerlo? Tenemos un problema.

Quizá se objetará que hay hombres que por su naturaleza son incapaces de hacer lo preciso para salir de este estado; pero se puede responder, que la causa de esta degradación ha nacido de los individuos mismos y 
como consecuencia de los desórdenes de su vida. Si son culpables y si han perdido el dominio de sí mismos, suya es la culpa, por haber los unos cometido malas acciones, y pasado los otros el tiempo en medio de los placeres de la mesa y de excesos vergonzosos. Los actos repetidos, de cualquier género que sean, imprimen a los hombres un carácter que corresponde a estos actos, lo cual puede verse evidentemente por el ejemplo de todos los que se dedican a cualquier ejercicio o trabajo, pues llegan a poder consagrarse a ello constantemente. No saber que en todas materias los hábitos y las cualidades se adquieren mediante la continuidad de actos, es un error grosero propio de un hombre que no conoce ni siente absolutamente nada (Aristóteles, 2001, 85).

$\mathrm{Ni}$ virtudes ni vicios gozan del aplauso contemporáneo. No lo hacen ni en el fondo ni en la forma. Serían, en el decir de López Quintás, términos antitalismán, cargados de connotación peyorativa (López Quintás, 1988). Serían, también, siguiendo a Alejandro Llano términos desgraciadamente desterrados, que habría que recuperar cuanto antes fomentando «ambientes fértiles, estimulantes, donde el vicio no se exalte ni se ridiculice la virtud; donde abunden las personalidades maduras [...] porque carácter y comunidad están vinculados [...] mi autorrealización exige un continuo diálogo con otras personas que en cierto modo forman parte de mí y de las que yo formo parte» (Llano, 2002, 38). ¡Qué complicado es encontrar este carácter de verdadera comunidad en el ámbito laboral! Y sin embargo, iqué necesario resulta!, porque la comunidad si es tal será siempre abierta, estará dirigida con sentido a un fin y no sucumbirá ahogada en los medios (digitales) que son imprescindibles, pero son tan solo instrumentos.

Necesitamos equilibrio, término medio. Necesitamos salir de la omnipresente conexión, escapar de tanto cansancio. Descansar, desconectar. Porque el exceso del aumento de rendimiento provoca el infarto del alma (Han, 2012,72). Y no hay aviso de salud más serio que ese, ni fundamento ético más sólido para cimentar el derecho a la desconexión, que trate de aliviar la distancia que existe entre nuestro ser y nuestro deber ser digital.

\section{Bibliografía}

Alter, A. (2018). Irresistible. ¿Quién nos ha convertido en yonquis tecnológicos? Barcelona: Paidós.

Aristóteles (2001). Ética a Nicómaco. Introducción, traducción y notas de José Luis Calvo Martínez. Madrid: Alianza Editorial.

Atienza, M. y Ruíz Manero, J. (2007). «Dejemos atrás el positivismo jurídico». Revista ISONOMÍA, n. 27, 7-28.

Bauman, Z. (2007). Tiempos líquidos. Barcelona: Tusquets.

Catela, I. (2012). Hijos conectados. Madrid: Palabra.

- (2018). Me desconecto, luego existo. Propuestas para sobrevivir a la adicción digital. Madrid: Encuentro.

Delgado, E. (2017). «Adicción a Internet: desmontando una mentira». En Lluna, S. y Pedreira, J., Los nativos digitales no existen. Cómo educar a tus hijos para un mundo digital. Barcelona: Deusto (69-87).

Eco, U. (1995). Apocalípticos e integrados. Barcelona: Tusquets.

Esquirol, J. M. (2015). La resistencia íntima. Ensayo de una filosofía de la proximidad. Barcelona: Acantilado. 
García Morente, M. (2011). Ensayo sobre la vida privada. Madrid: Encuentro.

Habermas, J. y Ratzinger, J. (2008). Entre razón y religión. Dialéctica de la secularización. México DF: Fondo de Cultura Económica.

Han, B.-C. (2012). La sociedad del cansancio. Barcelona: Herder.

- (2013). La sociedad de la transparencia. Barcelona: Herder.

- (2014). En el enjambre. Barcelona: Herder.

- (2014). Psicopolítica. Barcelona: Herder.

- (2018). Hiperculturalidad. Barcelona: Herder.

- (2018). La expulsión de lo distinto. Barcelona: Herder.

Lanier, J. (2011). Contra el rebaño digital. Un manifiesto. Barcelona: Debate.

- (2018). Diez razones para borrar tus redes sociales de inmediato. Barcelona: Debate.

Le Breton, D. (2016). Desaparecer de sí. Una tentación contemporánea. Madrid: Siruela.

Levinas, E. (2012). Totalidad e infinito. Salamanca: Sígueme.

López Quintás, A. (1988). Estrategias del lenguaje y manipulación del hombre. Madrid: Narcea.

- (2006). Vértigo y éxtasis: una clave para superar las adicciones. Madrid: Palabra.

Llano, A. (2002). La vida lograda. Barcelona: Ariel.

Malagón-Amor, Á., Córcoles Martínez, D., Martín-López, L. M. y Pérez-Solá, V. (2015). «Hikikomori in Spain: A descriptive study». International Journal of Social Psychiatry, vol. 61(5), 475-483.

Miller, J. A. (2013). Extimidad. Barcelona: Paidós.

Rodríguez Duplá, L. (2005). Ética de la vida buena. Bilbao: Desclée de Brower.

Saito, T. (2013). Hikikomori: Adolescence without End, Minnesota: University of Minnesota Press.

Sánchez-Carbonell, X. y Beranuy, M. (2007). «La adicción a internet como sobreadaptación social». En

A. Talarn (comp.)., Globalización y salud mental. Barcelona: Herder, 341-367.

Spitzer, M. (2013). Demencia digital. El peligro de las nuevas tecnologías. Barcelona: Ediciones B.

Suler, J. (1996). "Why is this eating my life? Computer and cyberspace addiction at the Palace», citado por J. A. García del Castillo et al. (2007) en Uso y abuso de internet en jóvenes universitarios. Recuperado a 6 de mayo de 2018 de http://www1.rider.edu/ suler/. 